Research Paper

\title{
E-cigarette aerosols induce unfolded protein response in normal human oral keratinocytes
}

Eoon Hye Ji1 ${ }^{*}$, Naseim Elzakra ${ }^{*}$, Wei Chen ${ }^{1}$, Li Cui ${ }^{1}$, Eon S Lee ${ }^{2}$, Bingbing Sun ${ }^{3,4}$, Diana Messadi ${ }^{1}$, Tian Xia ${ }^{3}$, Yifang $\mathrm{Zhu}^{2}$, Shen $\mathrm{Hu}^{1}{ }^{\circledR}$

1. School of Dentistry, University of California, Los Angeles, CA 90095, USA

2. Fielding School of Public Health, University of California, Los Angeles, CA 90095, USA

3. Division of Nanomedicine, School of Medicine, University of California, Los Angeles, CA 90095, USA

4. State Key Laboratory of Fine Chemicals, School of Chemical Engineering, Dalian University of Technology, 2 Linggong Road, 116024, Dalian, China

${ }^{*}$ Equal contribution

$\triangle$ Corresponding author: Shen Hu, PhD, MBA; Professor, UCLA School of Dentistry and Jonsson Comprehensive Cancer Center. 10833 Le Conte Ave, Los Angeles, CA 90095. Email: shenhu@ucla.edu; Phone: 310-2068834

(c) The author(s). This is an open access article distributed under the terms of the Creative Commons Attribution License (https://creativecommons.org/licenses/by/4.0/). See http://ivyspring.com/terms for full terms and conditions.

Received: 2018.11.07; Accepted: 2019.04.20; Published: 2019.11.19

\begin{abstract}
Objective: Since the introduction in 2004, global usage of e-cigarettes (ECs) has risen exponentially. However, the risks of ECs on oral health are uncertain. The purpose of this study is to understand if EC aerosol exposure impacts the gene pathways of normal human oral keratinocytes (NHOKs), particularly the unfolded protein response (UPR) pathway.

Materials and methods: EC aerosols were generated reproducibly with a home-made puffing device and impinged into the culture medium for NHOKs. DNA microarrays were used to profile the gene expression changes in NHOKs treated with EC aerosols, and the Ingenuity Pathway Analysis (IPA) was used to reveal signaling pathways altered by the EC aerosols. Quantitative PCR was used to validate the expression changes of significantly altered genes.

Results: DNA microarray profiling followed by IPA revealed a number of signaling pathways, such as UPR, cell cycle regulation, TGF- $\beta$ signaling, NRF2-mediated oxidative stress response, PI3K/AKT signaling, NF-KB signaling, and HGF signaling, activated by EC aerosols in NHOKs. The UPR pathway genes, C/EBP homologous protein (CHOP), activating transcription factor 4 (ATF4), $X$ box binding protein $1(\mathrm{XBPI})$, and inositol-requiring enzyme 1 alpha (IREI $\alpha)$ were all significantly up-regulated in EC aerosol-treated NHOKs whereas immunoglobulin heavy-chain binding protein (BIP) and PRKR-like ER kinase (PERK) were slightly up-regulated. QPCR analysis results were found to be well correlated with those from the DNA microarray analysis. The most significantly changed genes in EC aerosol-treated NHOKs versus untreated NHOKs were CHOP, ATF4, XBPI, IREI $\alpha$ and BIP. Meanwhile, Western blot analysis confirmed that CHOP, GRP78 (BIP), ATF4, IREla and XBPIs (spliced XBPI) were significantly up-regulated in NHOKs treated with EC aerosols.

Conclusion: Our results indicate that EC aerosols up-regulate the UPR pathway genes in NHOKs, and the induction of UPR response is mediated by the PERK - EIF2 $\alpha$ - ATF4 and IREl $\alpha$ - XBPI pathways.
\end{abstract}

Key words: E-cigarette aerosols, protein response, human oral keratinocytes

\section{Introduction}

Electronic cigarettes (ECs), also known as vapor cigarettes or vaporizer pens, are battery-powered devices that provide an inhalant containing nicotine and other additives. Most of the ECs are made with a heating element which generates aerosols from a E-liquid solution at a temperature of roughly 100 - 
$250^{\circ} \mathrm{C}$ [1]. Recently, there has been a dramatic increase in EC usage, mainly because ECs deliver nicotine with flavorings to users in vapor instead of smoke, which is much less prohibited in public areas. Many have switched from conventional cigarettes believing ECs had little or no risk [2]. Based on a survey study, about 2.4 million middle and high school students in the US are exposed to ECs. Strikingly, among the US high school students, the use of ECs has been increased from $1.5 \%$ to $13.4 \%$ based on a recent report [3]. Despite the consideration of ECs as a potential substitute smoking device for conventional smoking, many studies have investigated the behavior of EC users or pathological symptoms. However, these studies were mainly based on a short-term EC use [2, 4]. Generating data from long-term EC use is necessary to establish guidelines and regulatory decisions on EC production and usage.

Few studies have been carried out to investigate the effect of ECs on the oral cavity. Most of the current studies, in regards to EC research at the molecular level, are focused on relationships between EC and lung/airway epithelia cells or tissues. Lerner et al., stated that when lung cells and tissues were exposed to EC aerosols, oxidative and inflammatory responses occurred [5]. Sussan et al. demonstrated that, when mice were infected with Streptococcus pneumonia and exposed to EC aerosols, their pulmonary bacterial clearance was impaired significantly compared to air-exposed mice [6]. EC aerosols allow lung epithelia cells to be very susceptible to viral infections and cause weakened immune system. A recent study showed that exposure to EC aerosol mixtures with flavorings increased oxidative/carbonyl stresses and inflammatory cytokine release in human periodontal ligament fibroblasts, human gingival epithelium progenitors, and 3D EpiGingival tissues [7]. In our previous study, we characterized EC aerosols using a combination of advanced technologies. Our findings suggested that EC aerosols induce cytotoxicity to oral epithelial cells in vitro, which may be partially mediated by oxidative stress response [8]. Oxidative stress response may lead to numerous changes in the cells including gene expression changes depending on the level of oxidative stress. To the best of our knowledge, there has not been systematic analysis of the gene expression changes in oral epithelial cells caused by EC aerosols.

In this study, we have investigated the effects of EC aerosols on gene expression changes in normal human oral keratinocytes (NHOKs) and found that UPR is significantly activated by EC aerosols. In response to a variety of pathological stimuli, such as aerosols from cigarettes and possibly EC, nutrient deprivation, oxidative stress, DNA damage, or energy perturbation or fluctuations [9], endoplasmic reticulum (ER) stress may occur in eukaryotic cells and result in unfolded or misfolded proteins. This leads to the activation of a cascade of intracellular signaling molecules of the UPR pathway. UPR plays important role in restoring homeostasis, degrading misfolded proteins, and triggering cellular signals to assist protein folding. However, it may also lead to apoptosis if protein misfolding is not fixed [10]. Through DNA microarray and qPCR/Western blot analyses, we have demonstrated that EC aerosols activate the molecular determinants of the UPR signaling pathway in NHOKs.

\section{Materials and Methods}

\section{Cell culture}

NHOKs were maintained at $37{ }^{\circ} \mathrm{C}$ in an atmosphere of $95 \%$ air and $5 \% \mathrm{CO}_{2}$. The cells were passaged at $60-70 \%$ confluency level and allowed to proliferate in the EpiLife medium supplemented with the human keratinocyte growth supplement (Invitrogen, Carlsbad, CA), as described previously(Ji et al., 2016). When the NHOKs reached 60-70\% confluence, the cells were washed once with PBS and subsequently were incubated with the EC aerosol-impinged EpiLife culture media in the $\mathrm{CO}_{2}$ incubator. Generation of EC aerosols and EC aerosol-impinged cultured media are described below in details.

\section{Generation of EC aerosols}

EC aerosols were generated from an e-liquid mixture using an EC emission apparatus (Figure 1). The e-liquid mixture was prepared from individual chemical compounds of propylene glycol (PG, $\mathrm{C}_{3} \mathrm{H}_{8} \mathrm{O}_{2}, \geq 99.5 \%$ ), vegetable glycerin (VG, $\mathrm{C}_{3} \mathrm{H}_{8} \mathrm{O}_{3}, \geq 99.5 \%$ ), and nicotine (Nic, $\mathrm{C}_{10} \mathrm{H}_{14} \mathrm{~N}_{2}, \geq 99 \%$ ). The e-liquid mixture used in this study had a $29.3 \%$ PG and $68.3 \%$ VG with $2.4 \mathrm{mg}$ Nic/1. With the e-liquid mixture, e-cig aerosol emissions were generated by using a third generation EC device, so-called "Mods" (i.e., Vapor-fi model Volt Hybrid Tank used in this study). This type of EC device is selected because of its high popularity among the EC devices used.EC aerosols were generated with a thermal heating coil $(0.5 \Omega)$ in the EC device at a constant $7.5 \mathrm{~W}$ electrical power. Particle-free (i.e., HEPA-filtered) air was supplied to the EC device at $11 / \mathrm{min}$ airflow rate. The generated EC aerosols were collected in a series of three glass impingers. The impinged EC aerosol concentration per $1 \mathrm{ml}$ of medium used was: $14.89 \mathrm{mg}$ EC aerosol per $\mathrm{ml}$ medium. High throughput dynamic light scattering (HT-DLS, Dynapro ${ }^{\mathrm{TM}}$ Plate Reader, Wyatt Technology) was performed to determine the particle size and size distribution of the 
EC aerosols in aqueous solution. Transmission electron microscopy (TEM, JEOL 1200 EX, accelerating voltage $80 \mathrm{kV}$ ) was used to determine the morphology and primary size of EC aerosol nanoparticles.

\section{Treatment of NHOKs with EC aerosols}

EC aerosols were prepared as described above and immediately impinged into the NHOK culture media during 15 minutes. The particle suspensions were sonicated for 5 min using a water bath sonicator to obtain well-dispersed particle suspensions. Afterwards, the impinged culture medium was immediately used to treat NHOKs. After the NHOKs (on petri dish, $\sim 80 \%$ confluence) were washed once with PBS, the impinged culture medium was added to the petri dish and incubated with the cells for 4 hours $\left(5 \% \mathrm{CO}_{2}, 37{ }^{\circ} \mathrm{C}\right)$ prior to harvesting for DNA microarray and qPCR analyses.

\section{DNA microarray analysis}

RNA was extracted using the Qiagen RNAeasy Micro Kit, following the manufacturer's instruction. RNA purity/concentration was determined using a Nanodrop 8000 (Thermo Fisher, Waltham, MA), and RNA integrity was analyzed using an Agilent 2100 Bioanalyzer (Agilent Technologies, Palo Alto, CA). Microarray targets were generated using the FL-Ovation cDNA Biotin Module V2 (NuGen Technologies, San Carlos, CA) and then hybridized to the Affymetrix Gene Chip U133Plus 2.0 Array (Affymetrix, Santa Clara, CA), which contains > 54,000 probe sets representing $>47,000$ transcripts and variants, according to the manufacturers' instructions. The arrays were washed and stained with streptavidin phycoerythrin in Affymetrix GeneChip protocol, and then scanned using an Affymetrix GeneChip Scanner 3000. Microarray analysis was performed in duplicates and the AGCC software (Affymetrix) was utilized for acquisition of array images and initial quantification.

\section{Quantitative real-time PCR}

Total RNA was extracted from cultured cells using the RNeasy Mini Kit (Qiagen, Valencia, CA). cDNA conversion was carried out using Superscript II reverse transcriptase (Invitrogen), and $5 \mu \mathrm{g}$ of RNA per sample was converted. mRNA and cDNA concentration was measured using a Nanodrop spectrometer (Thermo Fisher). cDNA concentration of test and control samples was diluted to equal concentration $(100 \mathrm{ng} / \mu \mathrm{l})$. For qPCR reaction, $1 \mu \mathrm{l}$ of diluted cDNA solution was mixed with $0.4 \mu 1$ primer, 10 $\mu$ l DEPC-treated RNAase/DNA free water and 8.6 1 l SYBR Green I MasterMix (Roche, Indianapolis, IN) in a 96-well PCR plate and the reaction was performed on a CFX96 qPCR system (Bio-Rad, Hercules, CA). Actin was used as a housekeeping Gene for normalization. Three biological replicates were analyzed with qPCR and the data was analyzed using the $2^{-\Delta \Delta \mathrm{Ct}}$ method. Table 1 lists the sequences for the primers used in this study for qPCR analysis.

Table 1. The primers used for qPCR analysis in this study.

\begin{tabular}{ll}
\hline BIP F & TGCTGGCCTAAATGTTATG \\
BIP R & TGGTGAGAAGAGACACATC \\
PERK F & CTTATGCCAGACACACAGGACAA \\
PERK R & TCCATCTGAGTGCTGAATGGAATAC \\
IRE1a F & GAAGATCCAGTCCTGCAGGTC \\
IRE1a R & AGAAGAGAGGTTGATGGGCAG \\
XBP1 F & GTGAGCTGGAACAGCAAGTGGT \\
XBP1 R & CCAAGCGCTGTCTTAACTCCTG \\
ATF6 F & GCCGCCGTCCCAGATATTA \\
ATF6 R & GCAAAGAGAGCAGAATCCCA \\
CHOP F & TGCTTTCAGGTGTGGTGATGTATG \\
CHOP R & AATCAGAGCTGGAACCTGAGGA \\
ATF4 F & AAGCCTAGGTCTCTTAGATG \\
ATF4 R & TTCCAGGTCATCTATACCCA \\
\hline
\end{tabular}

\section{Western blot analysis}

Protein samples were separated with a $4-12 \%$ Bis-Tris NuPAGE gel (Invitrogen) and transferred onto nitrocellulose membrane by the Trans-blot SD semi-dry transfer cell (Bio-Rad, Brea, CA, USA). The membranes were blocked in TBST buffer containing 5\% nonfat milk (Santa Cruz Biotech), and incubated with antibodies against human protein (anti-GRP78, anti-CHOP, anti-IRE1a, anti-XBP1, anti-ATF4 and anti-ATF6a) at a dilution of 1:500 (Santa Cruz Biotech) overnight, followed by HRP linked anti-mouse or anti-rabbit IgG (1:5000; GE Healthcare). The detection was performed with the ECL-Plus Western blotting reagent kit (GE Healthcare). Western blot analysis was performed in triplicates.

\section{Statistical analysis}

The data were expressed as the mean \pm standard deviation, and analyzed by the independent samples t-test using the MedCalc (MedCalc Software, Ostend, Belgium). $\mathrm{P}$ values $<0.05$ were considered as statistically significant. Differentially expressed genes were selected at $\geq 2$-fold difference and $\mathrm{P}<0.05$. Functional pathway analysis of the genes at significantly altered levels (derived from DNA microarray analysis) was performed with the Ingenuity Pathway Analysis (IPA, Qiagen). The Ingenuity Pathways Knowledge Base (IPKB) provided all the published known functions and interactions. Fischer's exact test was used to calculate a P-value to determine the significance of each canonical pathway. 

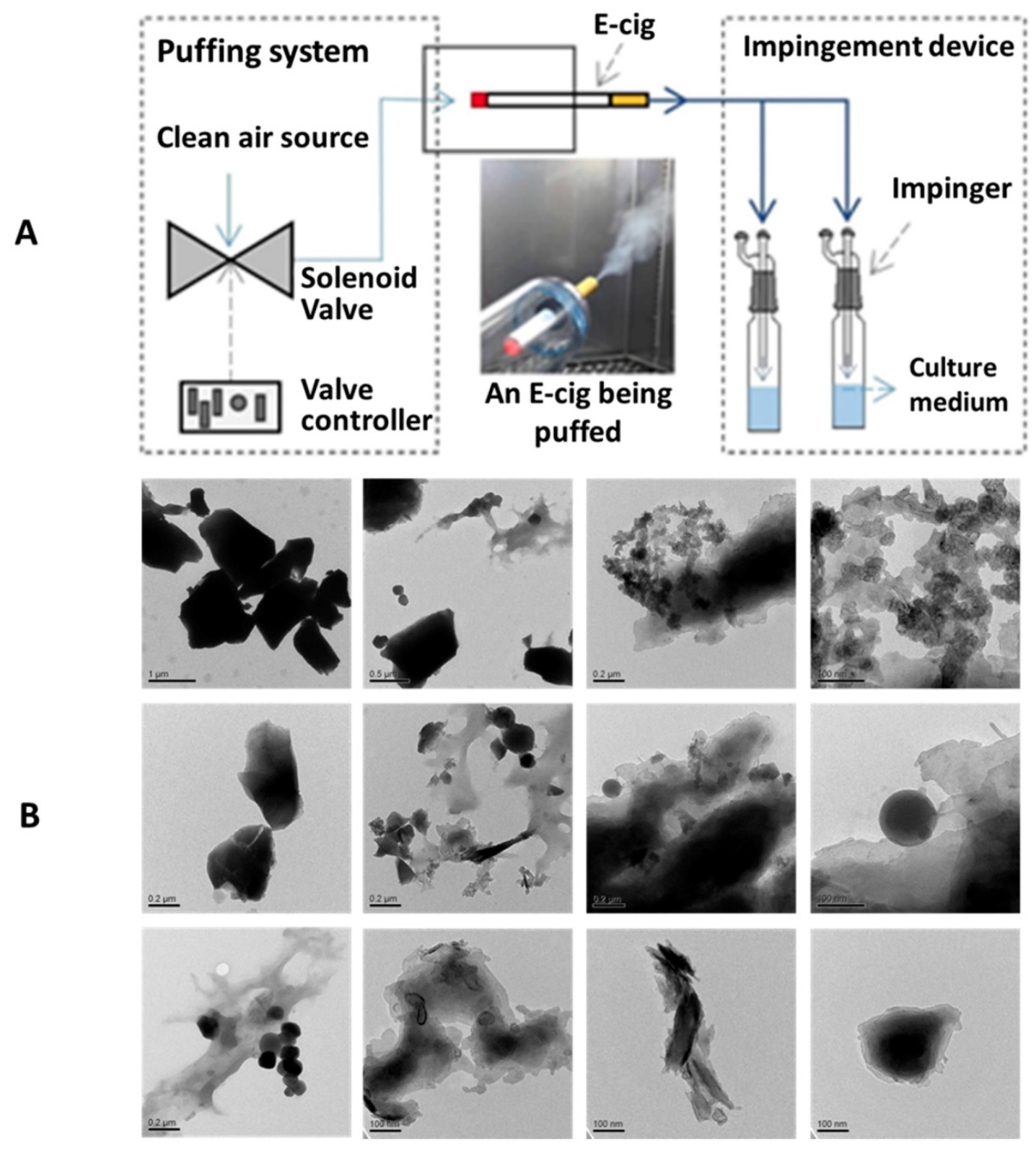

Figure 1. (A) A schematic diagram of the apparatus to generate EC aerosols and impinge the cell culture medium. (B) TEM images of EC aerosol microparticles/nanoparticles.

\section{Results}

\section{Generation of EC aerosols}

We have utilized a home-made EC puffing apparatus to generate the EC aerosols for in vitro experiments. As shown in Figure 1A, a puffing controller comprised of clean compressed air source, flow valve, and valve timer was used to push air through the tip and out of the mouth piece of an EC, to simulate reproducible EC aerosol delivery process. The generated EC aerosols were impinged into cell culture medium and, in 30 minutes, the impinged culture media were used to treat the NHOKs for 4 hours. Figure 1B shows the transmission electron microscope (TEM) image of EC aerosols, revealing the presence of flake-like and spherical micro-and nano-particles in EC aerosols. The size of EC aerosol nanoparticles in liquid phase varied significantly from $\sim 100 \mathrm{~nm}$ to $\sim 1 \mu \mathrm{m}$.

\section{DNA microarray analysis of EC aerosol- treated NHOKs}

We have profiled the gene expression changes in NHOKs treated with EC aerosols using the Human Genome U133 Plus 2.0 microarrays (Affymetrix). In total, 2350 genes were found to be significantly changed at 2-fold or more in EC aerosol-treated NHOKs when compared to untreated cells. Functional pathway analysis of the genes at significantly altered levels with the Ingenuity 
Pathway Analysis (IPA) indicated that many signaling pathways were activated in NHOKs due to the exposure to EC aerosols (Table 2). Among them, the UPR pathway was found to be ranked on the top. As shown in the UPR gene network generated by the IPA (Figure 2A), important molecular determinants of the UPR pathway such as activating transcription factor 4 (ATF4), C/EBP homologous protein (CHOP, a.k.a., DNA-damageinducible transcript 3), $\mathrm{X}$ box binding protein 1 (XBP1), and inositol-requiring enzyme 1 alpha (IRE1 $\alpha$, a.k.a., endoplasmic reticulum to nucleus signaling 1) were all activated in EC aerosol-treated NHOKs. Other types of signaling pathways such as TGF- $\beta$ and HGF were also activated in EC aerosol treated NHOKs (Figure 2B and 2C). TGF beta pathway is involved in many cellular processes such as cell growth, cell differentiation, and apoptosis whereas HGF pathway is related to cell proliferation, differentiation, and motility. A list of UPR pathway genes at differential expression levels between EC aerosol-treated and untreated NHOKs are shown in Table 3. CHOP (fold change, 9.60), ATF4 (fold change, 3.65), and XBP1 (fold change, 2.52) were most significantly changed genes. However, immunoglobulin heavy-chain binding protein (BIP, a.k.a., glucose-regulated protein 78 , fold change $=$ $1.68)$ and PRKR-like ER kinase (PERK, fold change $=$ 1.35) were modestly up-regulated whereas activating transcription factor 6a (ATP6a, fold change $=1.01)$ was almost unchanged.

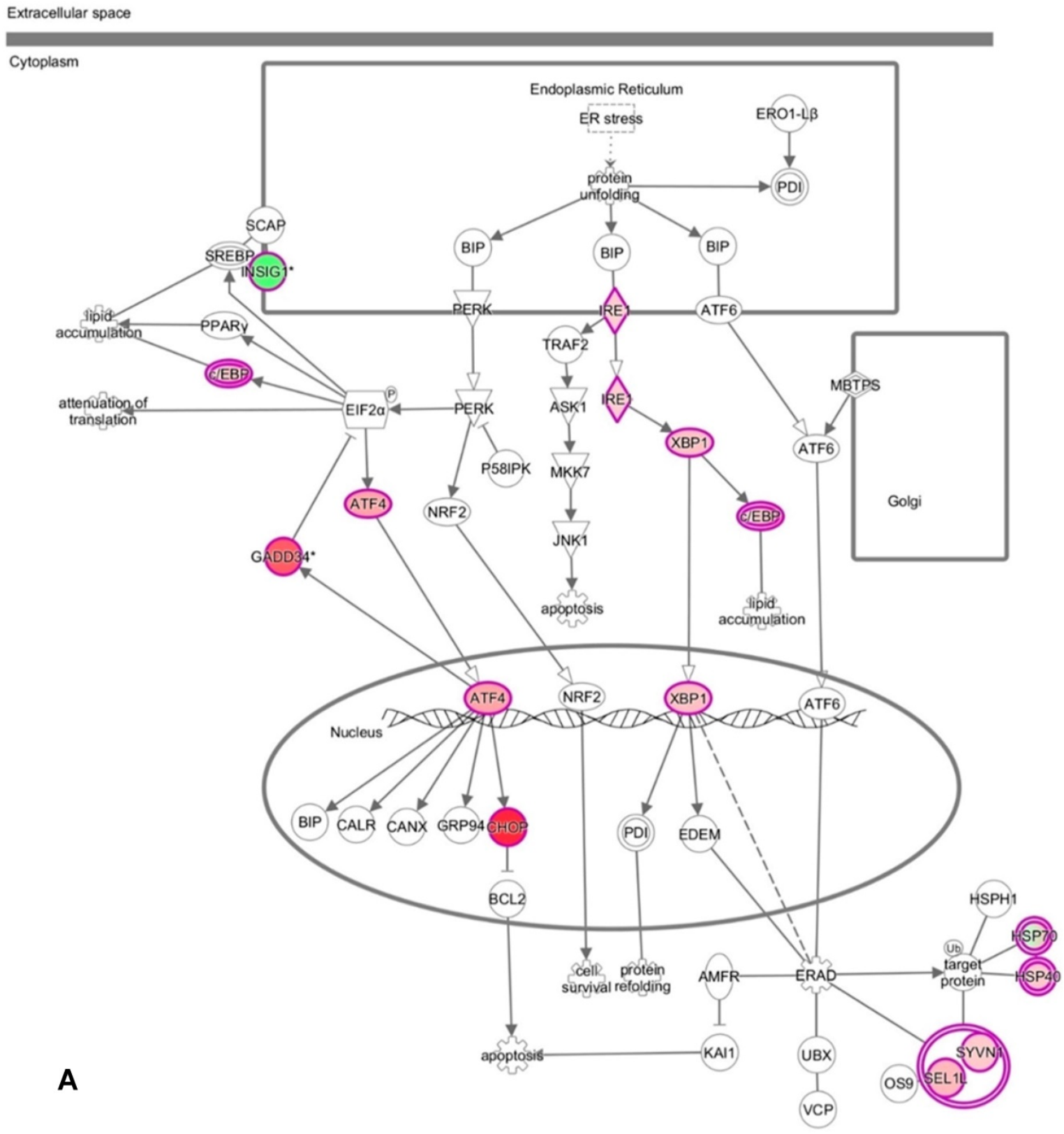



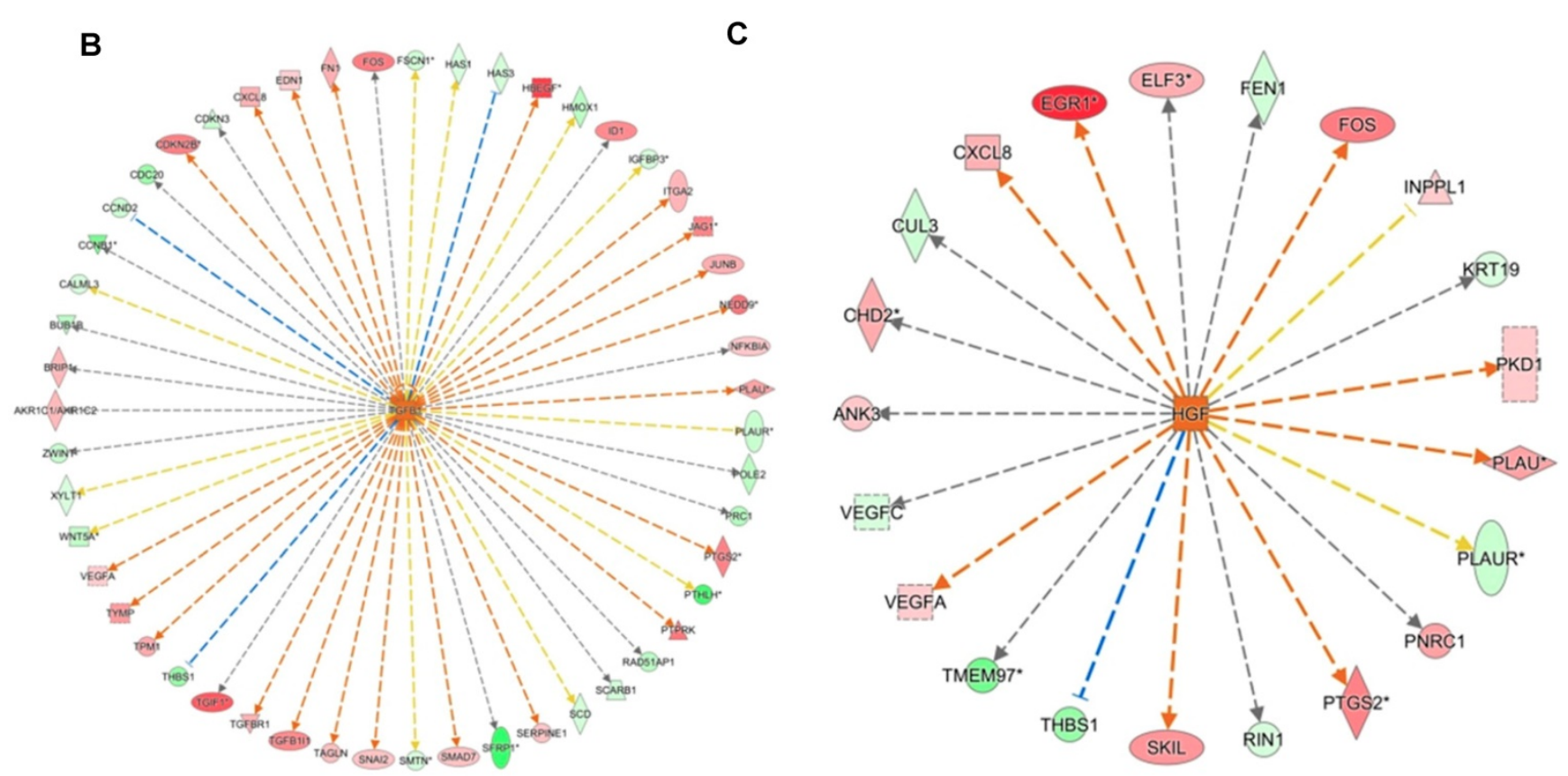

Figure 2. DNA microarray analysis followed by functional pathway analysis revealed unfolded protein response (UPR) pathways activated in normal human oral keratinocytes (NHOKs) by EC aerosols. (A) The canonical UPR pathway was generated with the Ingenuity Pathways Analysis (IPA) software. A number of genes of the UPR pathways such as XBP1 (X-box binding protein 1), IRE1 $\alpha$ (inositol-requiring enzyme 1, a.k.a., endoplasmic reticulum to nucleus signaling 1 ), ATF4 (activating transcription factor 4), CHOP (a.k.a., DDIT3, DNA-damage-inducible transcript 3 ) and C/EBP were induced by EC aerosols. (B \& C) TGF- $\beta$ and HGF signals activated in EC aerosol-treated NHOKs.

Table 2. Altered signaling pathways in NHOKs by EC aerosols.

\begin{tabular}{|c|c|c|c|}
\hline Ingenuity Canonical Pathways & $-\log (p)$ & $\begin{array}{l}\text { Number of } \\
\text { Molecules }\end{array}$ & Molecules \\
\hline Unfolded protein response & 5.48E00 & 16 & $\begin{array}{l}\text { DDIT3,HSPA14,ERN1,INSIG1,XBP1,CEBPB,DNAJB9,HSPA2,HSPA1L,CEBPG,HSPA8,SEL1L,SYVN1,HSP } \\
\text { A4,PPP1R15A,ATF4 }\end{array}$ \\
\hline Glucocorticoid Receptor Signaling & 4.25E00 & 42 & $\begin{array}{l}\text { ICAM1,TGFBR1,HSPA14,POU2F2,SOS2,GTF2H2,KRAS,HSPA1L,TSC22D3,IL1R2,HSPA4,IKBKB,GTF2B,N } \\
\text { FKBIA,NFAT5,HSP90AB1,ANXA1,TGFB2,FKBP5,SERPINE1,ADRB2,CREBZF,CXCL8,CDKN1C,NRAS,TA } \\
\text { F5L,CREBBP,SMARCE1,CEBPB,PPP3CC,STAT3,HSPA2,HSPA8,FOS,RRAS2,TAF5,IL1RN,HSP90AA1,NFA } \\
\text { TC2,IL1B,PLAU,PTGS2 }\end{array}$ \\
\hline Protein Ubiquitination Pathway & 3.62E00 & 38 & $\begin{array}{l}\text { B2M,USP24,CRYAB,USP45,HSPA14,CDC20,UBE2N,USP53,ANAPC10,CDC23,DNAJA1,HSPA1L,PAN2,H } \\
\text { SPA4,HSP90AB1,UBE2B,USP13,NEDD4L,PSMC2,NEDD4,USP15,USP9X,MDM2,HSPD1,DNAJB9,HSPA2, } \\
\text { HSPA12A,SKP2,USP31,HSPA8,USP32,CUL2,HSPA13,ANAPC5,HSP90AA1,DNAJB6,USP9Y,USP25 }\end{array}$ \\
\hline B Cell Receptor Signaling & 3.10E00 & 27 & $\begin{array}{l}\text { RAC2,POU2F2,SOS2,INPPL1,KRAS,BCL6,PTK2,IKBKB,NFKBIA,NFAT5,CFL2,ATF4,NRAS,PRKCQ,EGR1, } \\
\text { CREBBP,PPP3CC,ATF2,SYNJ2,CALM1 (includes } \\
\text { others),RRAS2,DAPP1,BCL10,PAG1,NFATC2,MAP2K3,MAP3K8 }\end{array}$ \\
\hline Molecular Mechanisms of Cancer & 2.98E00 & 47 & $\begin{array}{l}\text { FZD10,RAC2,BMP4,TGFBR1,FZD3,ARHGEF7,BMP2,SOS2,CTNNA1,KRAS,FZD1,CDKN2B,CCND1,PTK2, } \\
\text { NFKBIA,TGFB2,HIPK2,CDC25A,PMAIP1,NRAS,GNAS,PRKCQ,PAK6,TFDP1,CYCS,ITGA2,PTCH1,CREB } \\
\text { BP,SMAD7,CDK6,MDM2,AURKA,FZD8,FOS,CCNE1,CCND2,RRAS2,LEF1,FZD5,MAP2K3,CFLAR,BMP6, } \\
\text { NOTCH1,ARHGEF10,FNBP1,GNAL,WNT5A }\end{array}$ \\
\hline Cyclins and Cell Cycle Regulation & $2.86 \mathrm{E} 00$ & 15 & $\begin{array}{l}\text { HDAC9,HDAC4,TFDP1,PPP2CA,WEE1,CDK6,CCND1,CDKN2B,SKP2,CCNB1,CCNE1,CCND2,TGFB2,PP } \\
\text { P2R1B,CDC25A }\end{array}$ \\
\hline $\begin{array}{l}\text { Cell Cycle: G1/S Checkpoint } \\
\text { Regulation }\end{array}$ & $2.79 \mathrm{E} 00$ & 13 & HDAC9,HDAC4,TFDP1,CDK6,MDM2,CCND1,CDKN2B,SKP2,NRG1,CCNE1,CCND2,TGFB2,CDC25A \\
\hline TGF- $\beta$ Signaling & 2.74E00 & 16 & $\begin{array}{l}\text { BMP4,NRAS,TGFBR1,BMP2,CREBBP,SOS2,SMAD7,KRAS,TGIF1,INHBA,FOS,RRAS2,TGFB2,MAP2K3,SE } \\
\text { RPINE1,TFE3 }\end{array}$ \\
\hline ILK Signaling & 2.73E00 & 27 & $\begin{array}{l}\text { SNAI2,FN1,PPP2CA,BMP2,MYH11,RICTOR,CCND1,VEGFA,PTK2,NCK2,TGFB1I1,CFL2,PPAP2B,ATF4,I } \\
\text { RS2,ITGB5,NACA,CREBBP,FERMT2,VEGFC,ATF2,DOCK1,FOS,LEF1,PTGS2,PPP2R1B,FNBP1 }\end{array}$ \\
\hline PPAR Signaling & 2.58E00 & 16 & $\begin{array}{l}\text { NRAS,PPARD,CREBBP,SOS2,KRAS,IL1R2,IKBKB,FOS,IL18,NFKBIA,RRAS2,HSP90AB1,IL1RN,IL1B,HSP9 } \\
\text { 0AA1,PTGS2 }\end{array}$ \\
\hline $\begin{array}{l}\text { NRF2-mediated Oxidative Stress } \\
\text { Response }\end{array}$ & $2.55 \mathrm{E} 00$ & 26 & $\begin{array}{l}\text { NQO2,GCLC,KRAS,DNAJA1,CUL3,HMOX1,SCARB1,ATF4,GCLM,FKBP5,CBR1,NRAS,PRKCQ,NQO1,C } \\
\text { REBBP,HERPUD1,JUNB,DNAJB9,BACH1,FOS,RRAS2,STIP1,MAP2K3,SQSTM1,DNAJB6,ABCC4 }\end{array}$ \\
\hline Ephrin Receptor Signaling & 2.42E00 & 25 & $\begin{array}{l}\text { RAC2,PTPN13,SOS2,LIMK2,KRAS,VEGFA,PTK2,NCK2,GNB4,CFL2,EFNA5,ATF4,ACTR2,NRAS,GNAS,P } \\
\text { AK6,ITGA2,CREBBP,VEGFC,STAT3,EFNA1,ATF2,RRAS2,EPHB3,GNAL }\end{array}$ \\
\hline $\begin{array}{l}\text { Regulation of IL-2 Expression in } \\
\text { Activated and Anergic T Lymphocytes }\end{array}$ & 2.37E00 & 14 & $\begin{array}{l}\text { NRAS,TGFBR1,SOS2,KRAS,PPP3CC,IKBKB,CALM1 (includes } \\
\text { others),FOS,NFAT5,RRAS2,NFKBIA,BCL10,TGFB2,NFATC2 }\end{array}$ \\
\hline CDP-diacylglycerol Biosynthesis I & 2.12E00 & 5 & TAMM41,GPAM,AGPAT5,ABHD5,AGPAT9 \\
\hline $\begin{array}{l}\text { Regulation of the } \\
\text { Epithelial-Mesenchymal Transition } \\
\text { Pathway }\end{array}$ & 2.10E00 & 25 & $\begin{array}{l}\text { FZD10,ID2,SNAI2,TGFBR1,FZD3,SOS2,KRAS,FZD1,FGFR4,TGFB2,HMGA2,TWIST2,NRAS,JAG2,EGR1,ST } \\
\text { AT3,FZD8,RRAS2,LEF1,FZD5,MAP2K3,FGFRL1,JAG1,NOTCH1,WNT5A }\end{array}$ \\
\hline PI3K/AKT Signaling & $1.99 \mathrm{E} 00$ & 18 & $\begin{array}{l}\text { NRAS,PPP2CA,SOS2,ITGA2,MDM2,KRAS,INPPL1,CCND1,EIF4E,SYNJ2,IKBKB,NFKBIA,RRAS2,HSP90A } \\
\text { B1,HSP90AA1,MAP3K8,PTGS2,PPP2R1B }\end{array}$ \\
\hline
\end{tabular}




\begin{tabular}{|c|c|c|c|}
\hline $\begin{array}{l}\text { Human Embryonic Stem Cell } \\
\text { Pluripotency }\end{array}$ & 1.96E00 & 19 & $\begin{array}{l}\text { FZD10,TGFBR1,GNAS,BMP4,FZD3,BDNF,BMP2,SMAD7,FZD1,INHBA,S1PR3,FZD8,FGFR4,TGFB2,FZD5, } \\
\text { LEF1,FGFRL1,BMP6,WNT5A }\end{array}$ \\
\hline NF-kB Signaling & 1.93E00 & 23 & $\begin{array}{l}\text { AZI2,TGFBR1,BMP4,PRKCQ,NRAS,BMP2,UBE2N,CREBBP,TNFAIP3,KRAS,TANK,IL1R2,IKBKB,IL18,GH } \\
\text { R,RRAS2,NFKBIA,IL1RN,BCL10,FGFR4,IL1B,MAP3K8,FGFRL1 }\end{array}$ \\
\hline PAK Signaling & $1.90 \mathrm{E} 00$ & 14 & NRAS,PAK6,ARHGEF7,SOS2,ITGA2,KRAS,MYLK,LIMK2,PTK2,NCK2,RRAS2,CFL2,EPHB3,MYL12A \\
\hline Phosphatidylglycerol Biosynthesis II & $1.88 \mathrm{E} 00$ & 5 & TAMM41,GPAM,AGPAT5,ABHD5,AGPAT9 \\
\hline IL-6 Signaling & $1.85 \mathrm{E} 00$ & 17 & $\begin{array}{l}\text { CXCL8,SOCS3,NRAS,SOS2,KRAS,STAT3,CEBPB,VEGFA,IL1R2,FOS,IKBKB,IL18,RRAS2,NFKBIA,IL1RN,I } \\
\text { L1B,MAP2K3 }\end{array}$ \\
\hline IL-8 Signaling & $1.80 \mathrm{E} 00$ & 24 & $\begin{array}{l}\text { RAC2,CXCL8,NRAS,ICAM1,GNAS,PRKCQ,HBEGF,VEGFC,LIMK2,KRAS,PLD6,CCND1,PTK2,VEGFA,IK } \\
\text { BKB,HMOX1,FOS,GNB4,CCND2,RRAS2,PTGS2,FNBP1,ITGB5,IRAK2 }\end{array}$ \\
\hline Triacylglycerol Biosynthesis & 1.79E00 & 7 & GPAM,AGPAT5,ABHD5,PPP2R2D,PPAP2B,AGPAT9,ELOVL6 \\
\hline IL-10 Signaling & $1.65 \mathrm{E} 00$ & 11 & IL1R2,HMOX1,IKBKB,SOCS3,FOS,IL18,NFKBIA,IL1RN,IL1B,MAP2K3,STAT3 \\
\hline $\begin{array}{l}\text { Sphingosine and } \\
\text { Sphingosine-1-phosphate Metabolism }\end{array}$ & $1.64 \mathrm{E} 00$ & 3 & ASAH2B,NAAA,SGPP2 \\
\hline HGF Signaling & $1.63 \mathrm{E} 00$ & 15 & PRKCQ,NRAS,SOS2,ITGA2,KRAS,STAT3,CCND1,ATF2,PTK2,FOS,DOCK1,ELF3,RRAS2,MAP3K8,PTGS2 \\
\hline T Cell Receptor Signaling & $1.59 \mathrm{E} 00$ & 14 & $\begin{array}{l}\text { PRKCQ,NRAS,SOS2,KRAS,PPP3CC,IKBKB,CALM1 (includes } \\
\text { others),FOS,NFKBIA,NFAT5,RRAS2,BCL10,PAG1,NFATC2 }\end{array}$ \\
\hline p53 Signaling & 1.53E00 & 14 & $\begin{array}{l}\text { HDAC9,PMAIP1,GADD45B,TP63,SNAI2,PIAS1,MDM2,CCND1,CCNG1,CCND2,STAG1,THBS1,HIPK2,D } \\
\text { RAM1 }\end{array}$ \\
\hline PTEN Signaling & 1.48E00 & 16 & $\begin{array}{l}\text { RAC2,TGFBR1,NRAS,SOS2,ITGA2,INPPL1,KRAS,CCND1,SYNJ2,PTK2,IKBKB,MAGI1,RRAS2,GHR,FGFR } \\
\text { 4,FGFRL1 }\end{array}$ \\
\hline CD40 Signaling & $1.45 \mathrm{E} 00$ & 10 & TANK,IKBKB,FOS,ICAM1,NFKBIA,PTGS1,TNFAIP3,MAP2K3,STAT3,PTGS2 \\
\hline
\end{tabular}

Table 3. Differentially expressed genes of the UPR pathway between E-cig aerosol-treated and untreated NHOKs based on DNA microarray analysis.

\begin{tabular}{|c|c|c|c|}
\hline Gene \# & Protein name & Protein ID & $\begin{array}{l}\text { Average } \\
\text { fold change }\end{array}$ \\
\hline HSPA5 & $\begin{array}{l}\text { Immunoglobulin heavy-chain binding } \\
\text { protein (a.k.a., glucose-regulated protein } \\
78 \text { ) }\end{array}$ & $\begin{array}{l}\text { BIP } \\
\text { (GRP78) }\end{array}$ & 1.68 \\
\hline ERN1 & $\begin{array}{l}\text { Inositol-requiring enzyme } 1 \text { (a.k.a., } \\
\text { endoplasmic reticulum to nucleus } \\
\text { signaling 1) }\end{array}$ & IRE1a & 1.97 \\
\hline XBP1 & X-box binding protein 1 & XBP1 & 2.52 \\
\hline EIF2AK3 & PRKR-like ER kinase & PERK & 1.35 \\
\hline ATF6 & Activating transcription factor $6 a$ & ATF6a & 1.01 \\
\hline ATF4 & Activating transcription factor 4 & ATF4 & 3.65 \\
\hline DDIT3 & $\begin{array}{l}\text { C/EBP homologous protein (a.k.a., } \\
\text { DNA-damage-inducible transcript } 3 \text { ) }\end{array}$ & $\mathrm{CHOP}$ & 9.60 \\
\hline
\end{tabular}

\section{qPCR analysis of UPR pathway genes in EC aerosol-treated NHOKs}

Figure 3 shows the qPCR analysis results of the gene expression levels of CHOP, XBP1, ATF4, IRE1 $\alpha$, BIP, PERK and ATF6 $\alpha$ in NHOKs treated with EC aerosols in relative to untreated NHOKs. The most significantly changed genes were $\mathrm{CHOP}$ (fold change, 43.1), ATF4 (fold change, 14.3) and XBP1 (fold change, 4.42) according to the qPCR analysis. Meanwhile, PERK was slightly up-regulated (fold change, 1.56) whereas ATF6 $\alpha$ was not significantly changed. However, BIP was found to be significantly overexpressed (fold change, 3.29) in EC aerosol-treated NHOKs compared to untreated NHOKs. As shown in Table 3 and Figure 3, DNA microarray and qPCR analyses showed consistency on the expression changes of all genes except BIP. DNA microarray analysis indicated that BIP was modestly up-regulated in EC aerosol-treated cells but the gene was found to be significantly up-regulated by qPCR analysis. Nevertheless, these results confirmed that
EC aerosols activate the expression of UPR pathway genes in NHOKs.

\section{Western blot analysis of UPR pathway gene products in EC aerosol-treated NHOKs}

Figure 4 shows the Western blot analysis of GRP78 (BIP), CHOP, XBP1s (spliced XBP1), ATF4, IRE1 $\alpha$, and ATF6 $\alpha$ in EC aerosol-treated versus untreated NHOKs. The results indicated that EC aerosol treatment caused significant changes in protein levels of GRP78 (fold change, 2.03), CHOP (fold change, 4.74), ATF4 (fold change, 2.67), XBP1s (fold change, 3.44), and IRE1a (fold change, 2.37). Meanwhile, ATF6 was not significantly up-regulated (fold change, 1.42). These results further confirmed that EC aerosols activate the expression of UPR pathway genes in NHOKs.

\section{Discussion}

Upon ER stress, unfolded and misfolded proteins bind and sequester BIP protein (a.k.a., GRP78), thereby activating the UPR. The UPR comprises three parallel signaling branches: PERK (EIF2AK) - eukaryotic translation initiation factor $2 \alpha$ (EIF2a) - ATF4, IRE1a - XBP1 and ATF6a. First step following the accumulation of unfolded proteins is the activation of BIP and its dissociation from the other membranous stress sensors: PERK, IRE1aand ATF6a [10]. Once the dissociation takes place, PERK, IRE1a and ATF6a become activated in a cascade manner. PERK phosphorylates EIF2a and in turn activates ATF4, which up-regulates multiple downstream target molecules, including CHOP. ATF4 activate ER stress response genes that are responsible for the antioxidant reaction and synthesis of the amino acids needed for cell survival [11]. ATF4 also activates transcription of $\mathrm{CHOP}$ which is required for ER-stress-mediated apoptosis both in vitro and in vivo 
$[12,13]$. In fact, CHOP inhibits BCL2, which is an anti-apoptotic protein. On the other hand, IRE1a activates XBP1 and in turn up-regulates the expression of protein disulfide isomerase (PDI), an enzyme that allows proteins to quickly find the correct arrangement of disulfide bonds in their fully folded state, and therefore acts to catalyze protein folding [14].
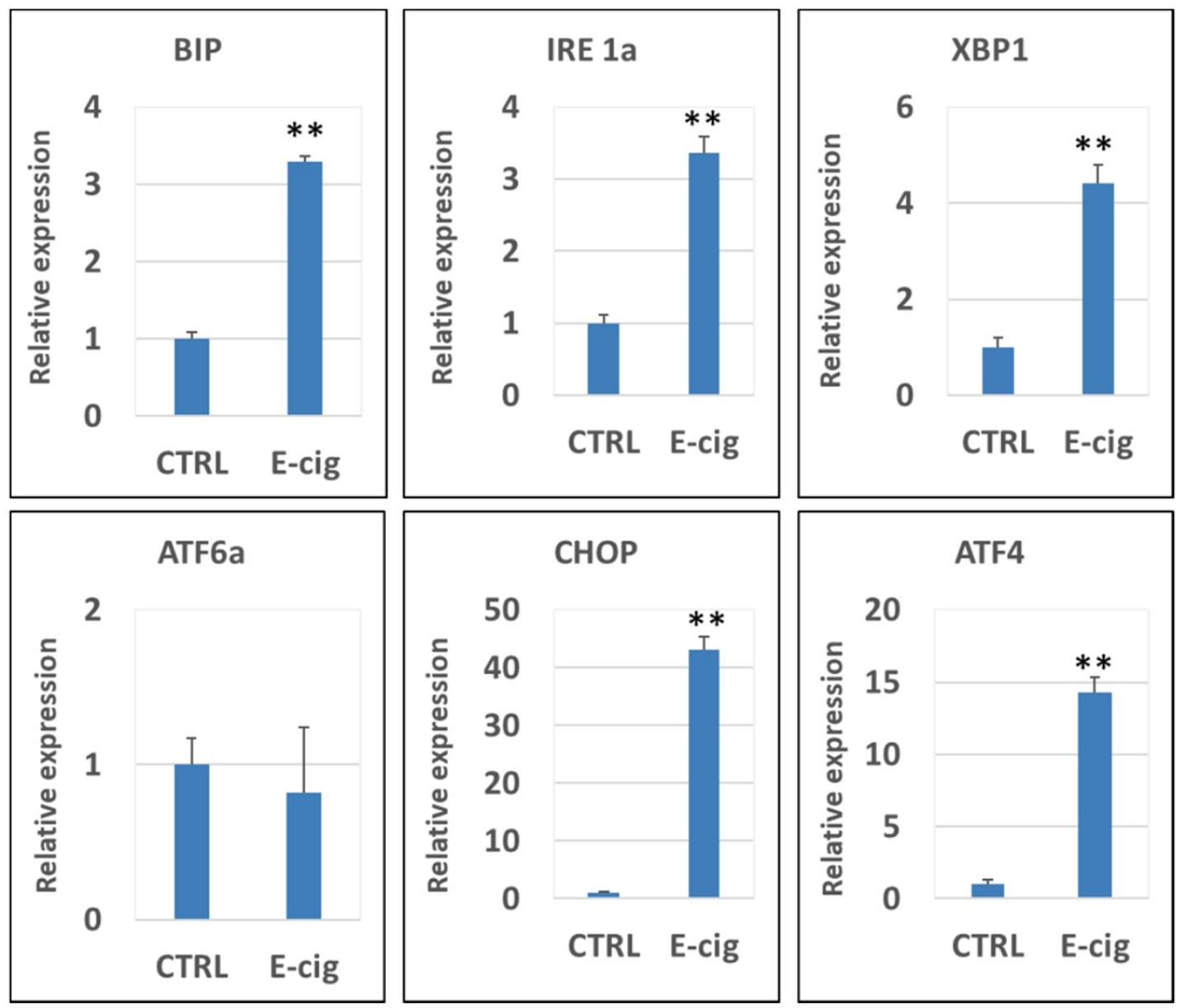

Figure 3. qPCR validation of gene expression of the UPR pathway in NHOKs treated with E-cig aerosols $(n=3)$. CHOP, ATF4, XBPI and IREl $\alpha$ and BIP were significantly up-regulated in NHOKs after the EC aerosol exposure (**, $p<0.01$; fold change $>3$ ). However, ATF6 was not significantly altered by EC aerosols. The primers used for qPCR analysis were listed in Table 1.
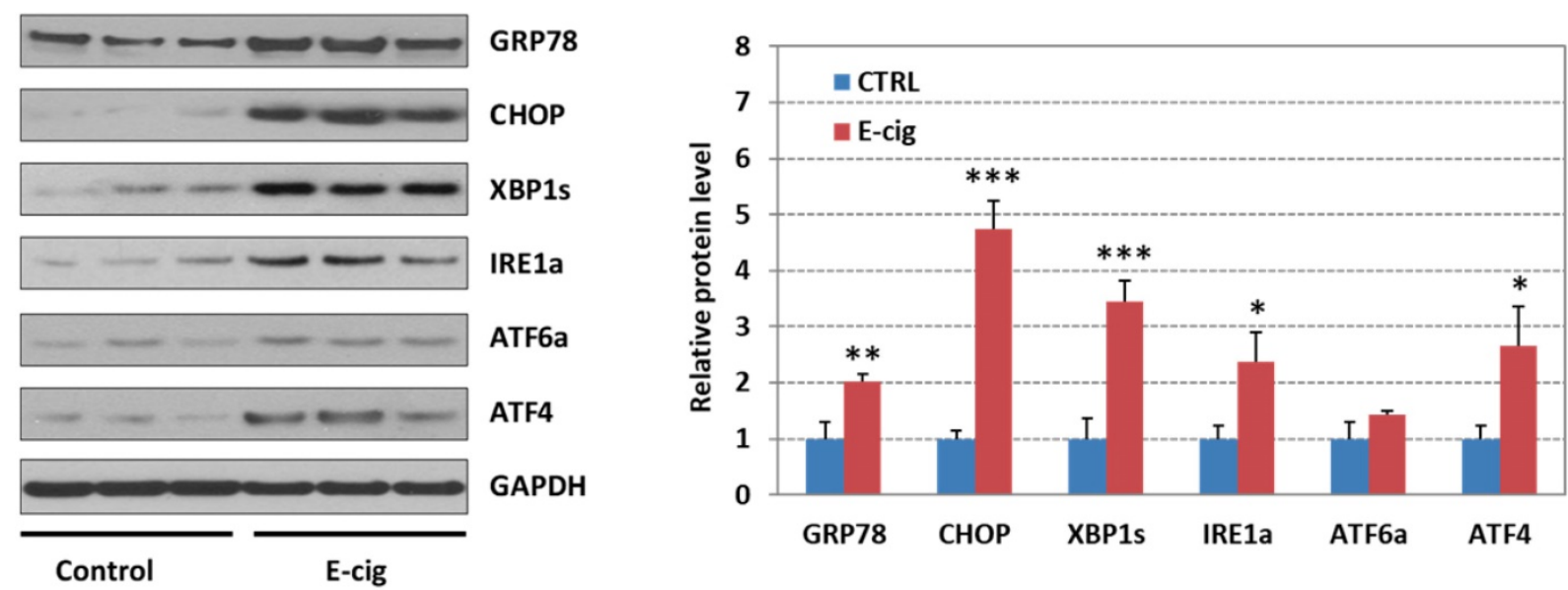

Figure 4. Western blot analysis of GRP78 (BIP), CHOP, IREIa, XBPIs (spliced XBPI), ATF4 and ATF6 in NHOKs treated with EC aerosols ( $n=3$, *, $p<0.05$; **, $\mathrm{p}$ $<0.01$ ). The results confirmed that GRP78, CHOP, IREI $\alpha$, XBPIs and ATF4 were significantly up-regulated in NHOKs by EC aerosols. 
The outcome of UPR activation increases protein folding, transport and ER-associated protein degradation (ERAD), while attenuating protein synthesis. Such pre-adaptive events, by blocking global protein synthesis, will eventually restore ER homeostasis $[15,16]$. However, if protein misfolding is not resolved, cells enter apoptosis. Induction of $\mathrm{CHOP}$ is an alternative element that becomes activated to switch from pro-adaptive to pro-apoptotic signaling when the damage is severe and prolonged [17, 18]. Under conditions of chronic stress, PERK activation leads to apoptosis, as the IRE1a - XBP1 and ATF6a pathways are attenuated [19-21]. Therefore, PERK activation promotes both adaptive and apoptotic responses depending on the severity of the stress. The stress response is most likely to be a tissue-specific reaction depending on the threshold of the ER stress tolerance of the tissue [19-21].

Our microarray study revealed a number of functional pathways in the NHOKs that may be altered by EC aerosols. These activated pathways include UPR, protein ubiquitination, oxidative stress response, NF-kB signaling, IL-6 signaling, IL-8 signaling, IL-10 signaling, TGF- $\beta$ signaling, HGF signaling, cell cycle regulation, EMT regulation, etc. Some of these pathways are related to cancer mechanisms or inflammation. Importantly, both microarray and qPCR analyses demonstrated that EC aerosols induce UPR in NHOKs. In fact, both datasets were well correlated with respect to the expression of UPR pathway genes. In response to EC aerosol treatment, $\mathrm{CHOP}, \mathrm{ATF} 4, \mathrm{XBP} 1$ (total expression) and IRE1a were found to be significantly over-expressed in NHOKs. Particularly, CHOP was dramatically up-regulated (fold change, 43.1). However, ATF6 $\alpha$ gene expression was almost not changed by EC aerosol exposure, as indicated by both microarray and qPCR analyses although its protein expression slightly increased. Whether ATF6 signals are involved in the activation of UPR by EC aerosols remains to be further verified. Western blot analysis also confirmed that GRP78 (BIP), CHOP, ATF4, XBP1s (spliced XBP1) and IRE1a were significantly up-regulated in NHOKs treated with EC aerosols. These results suggest that EC aerosols most likely induce UPR response via the mediation of PERK - EIF2 $\alpha$ - ATF4 and IRE1a - XBP1 signals. The dramatic over-expression of CHOP also implies that EC aerosols may cause apoptosis of NHOKs via the activation of cascade of PERK, EIF2a, ATF4 and CHOP. In fact, recent studies have demonstrated that conventional smoke induces UPR via PERK - EIF2a - ATF4 and IRE1a - XBP1 signals [22, 23].

In summary, our study has demonstrated that
EC aerosols induce the UPR response in normal human oral epithelial cells mediated by PERK - EIF2a - ATF4 and IRE1a - XBP1 signals. As mentioned earlier, UPR plays important role in restoring homeostasis and assisting protein folding in cells. However, it may also lead to apoptosis under chronic stress and cause cytotoxicity. What chemical components or physiochemical characteristics of EC aerosols induce the UPR signaling remains unknown. It may result from nanoparticles or trace amount of heavy metals present in EC aerosols [8]. Some other pathways related to cancer mechanisms or inflammation may also be activated in oral keratinocytes by EC aerosols although this needs to be further verified. EC is generally considered safer than conventional tobacco cigarettes; however, their impact on oral and systemic health remains uncertain. Considering the rapid increase of EC consumption especially among adolescents and young adults, it is important to study potential adverse effect of ECs on the oral health. It should be noted that our in vitro experimental setting may not well represent the actual in vivo condition for studying the effect of EC aerosols. Animal model studies as well as the investigation of cellular UPR pathways in EC users may confirm our in vitro study results and lead to additional findings. In addition, it is highly relevant to compare conventional cigarettes with ECs in terms of their induction of cellular stress responses (e.g., UPR and oxidative stress). These are valid concerns and certainly warrant further studies in the future.

\section{Acknowledgements}

This study was supported by the Tobacco-Related Disease Research Program (SH, 27IP-0058).

\section{Author contributions}

EHJ, NE and LC: Cell culture and treatment experiments, assays and data analysis. EL and YZ: Device puffing protocol and generation of $\mathrm{EC}$ aerosols. BS and TX: TEM characterization of EC aerosols. SH, YZ, TX and DM conceived the study, designed the experiments and wrote the manuscript.

\section{Competing Interests}

The authors have declared that no competing interest exists.

\section{References}

1. Rowell TR, Tarran R. Will chronic e-cigarette use cause lung disease? Am J Physiol Lung Cell Mol Physiol. 2015; 309: L1398-409.

2. Gualano MR, Passi S, Bert F, La Torre G, Scaioli G, Siliquini R. Electronic cigarettes: assessing the efficacy and the adverse effects through a systematic review of published studies. J Public Health (Oxf). 2015; 37: 488-97.

3. Singh T, Marynak K., Arrazola R.A., et al. Vital signs: exposure to electronic cigarette advertising among middle school and high school students - United States. Morbidity and Mortality Weekly Report. 2016; 64: 1403-8. 
4. Hua M, Alfi M, Talbot P. Health-related effects reported by electronic cigarette users in online forums. J Med Internet Res. 2013; 15: e59.

5. Lerner CA, Sundar IK, Yao H, Gerloff J, Ossip DJ, McIntosh S, et al. Vapors produced by electronic cigarettes and e-juices with flavorings induce toxicity, oxidative stress, and inflammatory response in lung epithelial cells and in mouse lung. PLoS One. 2015; 10: e0116732.

6. Sussan TE, Gajghate S, Thimmulappa RK, Ma J, Kim JH, Sudini K, et al Exposure to electronic cigarettes impairs pulmonary anti-bacterial and anti-viral defenses in a mouse model. PLoS One. 2015; 10: e0116861.

7. Sundar IK, Javed F, Romanos GE, Rahman I. E-cigarettes and flavorings induce inflammatory and pro-senescence responses in oral epithelial cells and periodontal fibroblasts. Oncotarget. 2016; 7: 77196-204.

8. Ji EH, Sun B, Zhao T, Shu S, Chang CH, Messadi D, et al. Characterization of Electronic Cigarette Aerosol and Its Induction of Oxidative Stress Response in Oral Keratinocytes. PLoS One. 2016; 11: e0154447.

9. McMillan DR, Gething MJ, Sambrook J. The cellular response to unfolded proteins: intercompartmental signaling. Curr Opin Biotechnol. 1994; 5: 540-5.

10. Szegezdi E, Logue SE, Gorman AM, Samali A. Mediators of endoplasmic reticulum stress-induced apoptosis. EMBO Rep. 2006; 7: 880-5.

11. Harding HP, Zhang $Y$, Bertolotti A, Zeng H, Ron D. Perk is essential for translational regulation and cell survival during the unfolded protein response. Mol Cell. 2000; 5: 897-904.

12. Marciniak SJ, Yun CY, Oyadomari S, Novoa I, Zhang $Y$, Jungreis R, et al. $\mathrm{CHOP}$ induces death by promoting protein synthesis and oxidation in the stressed endoplasmic reticulum. Genes Dev. 2004; 18: 3066-77.

13. Song B, Scheuner D, Ron D, Pennathur S, Kaufman RJ. Chop deletion reduces oxidative stress, improves beta cell function, and promotes cell survival in multiple mouse models of diabetes. J Clin Invest. 2008; 118: 3378-89.

14. Marcu MG, Doyle M, Bertolotti A, Ron D, Hendershot L, Neckers L. Heat shock protein 90 modulates the unfolded protein response by stabilizing IRE1alpha. Mol Cell Biol. 2002; 22: 8506-13.

15. Bertolotti A, Zhang Y, Hendershot LM, Harding HP, Ron D. Dynamic interaction of $\mathrm{BiP}$ and ER stress transducers in the unfolded-protein response. Nat Cell Biol. 2000; 2: 326-32.

16. Liu CY, Schroder M, Kaufman RJ. Ligand-independent dimerization activates the stress response kinases IRE1 and PERK in the lumen of the endoplasmic reticulum. J Biol Chem. 2000; 275: 24881-5.

17. Harding HP, Novoa I, Zhang Y, Zeng H, Wek R, Schapira M, et al. Regulated translation initiation controls stress-induced gene expression in mammalian cells. Mol Cell. 2000; 6: 1099-108.

18. Xu C, Bailly-Maitre B, Reed JC. Endoplasmic reticulum stress: cell life and death decisions. J Clin Invest. 2005; 115: 2656-64

19. Lin JH, Li H, Yasumura D, Cohen HR, Zhang C, Panning B, et al. IRE1 signaling affects cell fate during the unfolded protein response. Science. 2007; 318: 944-9.

20. Rutkowski DT, Arnold SM, Miller CN, Wu J, Li J, Gunnison KM, et al. Adaptation to ER stress is mediated by differential stabilities of pro-survival and pro-apoptotic mRNAs and proteins. PLoS Biol. 2006; 4: e374.

21. Scheuner D, Patel R, Wang F, Lee K, Kumar K, Wu J, et al. Double-stranded RNA-dependent protein kinase phosphorylation of the alpha-subunit of eukaryotic translation initiation factor 2 mediates apoptosis. J Biol Chem. 2006; 281: 21458-68.

22. Kunchithapautham K, Atkinson C, Rohrer B. Smoke exposure causes endoplasmic reticulum stress and lipid accumulation in retinal pigment epithelium through oxidative stress and complement activation. J Biol Chem. 2014; 289: 14534-46.

23. Wang $\mathrm{Y}, \mathrm{Wu} \mathrm{ZZ,} \mathrm{Wang} \mathrm{W}$. Inhibition of endoplasmic reticulum stress alleviates cigarette smoke-induced airway inflammation and emphysema. Oncotarget. 2017; 8: 77685-95. 